\title{
Researchers' Toolbox for the Future: Understanding and Designing Accessible and Inclusive Artificial Intelligence (AIAI)
}

\author{
Marianne Kinnula \\ INTERACT Research Unit, University \\ of Oulu, Finland \\ kinnula.marianne@oulu.fi \\ Grace Eden \\ Department of Human-Centered \\ Design, Indraprastha Institute of \\ Information Technology Delhi, India \\ Prema Nedungadi \\ Amrita Vishwa Vidyapeetham, India
}

\author{
Netta Iivari \\ INTERACT Research Unit, University \\ of Oulu, Finland \\ iivari.netta@oulu.fi
}

Markku Turunen

Faculty of Information Technology and Communication Sciences,

Tampere University, Finland

Tero Avellan

Tampere University, Tampere, Finland

Rucha Tulaskar

Tampere University, Tampere, Finland

\author{
Sumita Sharma \\ INTERACT Research Unit, University \\ of Oulu, Finland \\ sharma.sumita@oulu.fi
}

Krishnashree Achuthan

Amrita Vishwa Vidyapeetham, India

Biju Thankachan

Tampere University, Tampere, Finland

\begin{abstract}
As Artificial Intelligence (AI) is integrated into all things technical, there is a valid concern over its lack of diversity, inclusiveness, and accessibility. Further, questions such as what it means for AI to be accessible and inclusive, why is inclusive AI required, and how can it be achieved, is an emerging area of research. In this two-part workshop, we will explore the nuanced challenges towards Accessible and Inclusive AI together with participants with diverse backgrounds. First, we will collaboratively define Accessible and Inclusive AI (AIAI), building on the diverse experiences of the participants and moderators. The goal is to contribute to the formulation of a shared vision for Accessibility and AI as well as identify the challenges and opportunities towards realizing this vision. Working in small teams, participants will collaboratively conceptually design a future scenario for AIAI or critically analyse an example solution. The aim is for teams to tackle tough questions related to what it means for $\mathrm{AI}$ to be accessible and inclusive, while addressing algorithmic biases and limitations of AI, in addition to opportunities for overcoming them in the future. Finally, teams will present their conceptual designs and scenarios in the larger group. Overall, the workshop will ignite innovative, and even provocative, ideas and future scenarios, building towards an inclusive and accessible AI.
\end{abstract}

\section{CCS CONCEPTS}

- Human-centered computing $\rightarrow$ Accessibility; Accessibility theory, concepts and paradigms.

\section{KEYWORDS}

Empowerment, Accessibility, Inclusion, Artificial Intelligence

\section{ACM Reference Format:}

Marianne Kinnula, Netta Iivari, Sumita Sharma, Grace Eden, Markku Turunen, Krishnashree Achuthan, Prema Nedungadi, Tero Avellan, Biju Thankachan, and Rucha Tulaskar. 2021. Researchers' Toolbox for the Future: Understanding and Designing Accessible and Inclusive Artificial Intelligence (AIAI). In Academic Mindtrek 2021 (Mindtrek '21), fune 0103, 2021, Tampere/Virtual, Finland. ACM, New York, NY, USA, 5 pages. https://doi.org/10.1145/xxxxxxx.xxxxxxxx

\section{INTRODUCTION}

As Artificial Intelligence (AI) is integrated into all things technical, there is increasing concern over its lack of diversity, inclusiveness, and accessibility [1] [2] [11] [15]. However, questions such as what it means for AI to be accessible and inclusive, why is inclusive AI required, and how can it be achieved, is an emerging area of research. Further, for digital transformation of society to be empowering, inclusive, and accessible, it is important that researchers working in this field collaboratively bring these issues to the forefront and discuss them together with experts, students, and practitioners from diverse backgrounds. With this in mind, we propose a full day workshop on Accessible and Inclusive Artificial Intelligence (AIAI) with a two-folded approach.

First, we will collaboratively define in the workshop with participants asking the question: "what is Accessible and Inclusive AI?". We will achieve this by building on the diverse experiences of the participants and moderators. The goal is to contribute to the formulation of a shared vision for AIAI. Second, with the growing awareness of the algorithmic biases and limitations of AI, we will identify the challenges towards this vision as well as consider the
Permission to make digital or hard copies of all or part of this work for personal or
classroom use is granted without fee provided that copies are not made or distributed for profit or commercial advantage and that copies bear this notice and the full citation on the first page. Copyrights for components of this work owned by others than the author(s) must be honored. Abstracting with credit is permitted. To copy otherwise, or republish, to post on servers or to redistribute to lists, requires prior specific permission and/or a fee. Request permissions from permissions@acm.org.

Mindtrek '21, June 01-03, 2021, Tampere/Virtual, Finland

(c) 2021 Copyright held by the owner/author(s). Publication rights licensed to ACM. ACM ISBN $x x x-x-x x x x-x x x x-x$

https://doi.org/10.1145/xxxxxxx.Xxxxxxx 
opportunities and ways to overcome challenges in the future. This will be achieved using future-oriented methods [4] [14], such as design fiction, speculative design and other approaches to developing future scenarios, as they enable participants to consider out of the box solutions and approach the topics without focusing on current limitations.

The main goals of the workshop are to:

- Reflect on previous work in AIAI, through presentations by the workshop moderators.

- Build on the previous research and workshop participants' work, creating a shared vision for AIAI, with a focus on:

$\circ$ ethical aspects of AI with marginalized users, especially with regards to human agency

$\circ$ identifying the challenges and opportunities

$\circ$ devising future pathways and defining ways for monitoring progress

- creating an agenda for future research in this domain

- Provide participants an opportunity to experience using future-oriented approaches, that they can add to their 'methodological toolbox'.

- When exploring the future-oriented methods, examining how they can be adapted to designing accessible and inclusive AI applications, with a focus on advantages and limitations of these methods within the context of empowerment of marginalized users.

The workshop targets students, researchers, practitioners, and experts across the world who are interested in and working on accessibility, inclusion, and AI with diverse and marginalized users. Therefore, we also aim for the creation of a consortium of diverse experts and students, strengthening the research outreach in the field of AI, and accessibility and inclusion.

The workshop will be advertised on social networks (LinkedIn, Twitter, Facebook) and a call for papers (CfP) will be circulated to relevant professional mailing lists to recruit participants. The workshop webpage will include additional information, such as relevant readings on future-oriented methods to engage participants before the conference. Researchers' toolbox for the future is a series of workshops dedicated towards exploring, discussing, redefining, and formulating methods for imagining the future of technology, one that is empowering albeit provocative (http://interact.oulu.fi/researcherstoolbox..

\section{BACKGROUND}

As machines and software mimic, learn, and outgrow human decision making and understanding, Artificial Intelligence (AI) is already changing the world. AI is becoming more embedded in our everyday lives - such as access to services (e.g., banking, healthcare, transportation), decision making (recruitment and jobs, healthcare), and online and offline experiences. However, as an age old saying goes - "with great power comes great responsibility". It is no secret that current trends in AI may not be inclusive or accessible [2] [11], and that they inadvertently exacerbate existing digital divides, biases, and marginalization of entire communities and groups of people. For instance, there can be bias in the algorithms, such as when the assessment of people repeating a crime in the United
States was unfairly skewed towards minority groups [1], and Amazon's recruitment algorithm that mirrored gender bias in analysing resumes and unfairly denied capable female candidates [3]. Having diverse teams in the technology design can eliminate such discrimination, unethical use, and unfair practices [2] [15].

Additionally, there is a lack of representation of data from diverse users, this includes people with special needs and marginalized communities (e.g. inclusion). Representation of minorities and special groups is crucial for AI to be inclusive. Lastly, the application or service being designed should cater to diverse users' needs, regardless of disability by designing an application that ensures it is accessible by a larger group of users or by targeting the application towards a specific group of users (e.g. accessibility). For AI to be truly accessible and inclusive, changes are required at all three levels - the algorithm, the data, and the end-users [2]. Existing ethical frameworks address mainly individual, human responsibility, rather than distributed responsibility, which we argue is the need of the hour. Users of AI systems are also subject to ethical challenges such as inclusivity, bias, privacy, error, expectation setting, simulated data, and social acceptability. While some of these aspects may be addressed through legal regulation - in the future, this means that developers and designers need to understand the effects of AI on humans in order to be accessible and inclusive [11]. The AIAI workshop aligns itself with these concerns by creating awareness of these issues.

During the workshop, moderators and participants from diverse backgrounds and locations will come together. Workshop moderators will bring difficult conversations on AI to the forefront and open up the discussion to technical and nontechnical participants. This includes discussing European research principles and ethics, and attention and proactive stance on AI (e.g. EU directives on Trustworthy AI [5]), which gives us a head start on this topic. Within the workshop organizers, there is expertise in AI and ethics [2] [10], Human Centered AI [6], accessibility in India and Finland [8] [12], empowerment and inclusion [7] [9] diversity in IT [13] and studies have been conducted with Finnish school children to teach them digital technology design [7] [9], as well as basic AI principles. In addition, the work provides opportunities to engage with participants from diverse backgrounds and cultures.

Further, the AIAI workshop will leverage Global North and Global South experience and expertise together, to consider inclusive, accessible AI technology not as a privilege but a right. Focusing on students, academics, researchers, and industry allows us to have an impact on future and current technology designers and makers, and to train them as critical thinkers, asking tough questions, and striving for answers and solutions that make the world a better place for everyone.

\section{PLANNED STRUCTURE}

Before the workshop, participants submit a position paper, where they address the topic of AIAI: what is their personal vision for it and how they relate it to their own research, to orient them to the workshop topic. The participants are also given a reading package on the workshop topic. The workshop consists of two 2-hour sessions. Each session is a mix of expert presentations and handson activities (see the planned schedule in Table 1). In the expert presentations, participants are introduced to accessible, inclusive, 
Table 1: Planned Sessions Outline

\begin{tabular}{|c|c|}
\hline Session 1 (120 minutes) & Session 2 (120 minutes) \\
\hline $\begin{array}{l}15 \text { mins: Introduction to the workshop and agenda (UO) } \\
15 \text { mins: Empowering AI (expert presentation, UO) } \\
15 \text { mins: Participant introductions in small teams in breakout rooms } \\
\text { (UO) } \\
15 \text { mins: Human Centered AI (expert presentation, IIIT-D) } \\
30 \text { mins: Team activity \#1 in breakout rooms: } \\
\text { Discuss and consider: } \\
\text {-What is Accessible and Inclusive AI - how do we define it? } \\
\text {-What are some challenges identify the challenges towards this vision } \\
\text { as well as consider the opportunities and ways to overcome challenges } \\
\text { in the future? } \\
15 \text { mins: Discussion in larger group } \\
15 \text { mins: Introduction to future-oriented methods (expert presentation, } \\
\text { UO) }\end{array}$ & $\begin{array}{l}15 \text { mins: Accessible AI (expert presentation, TAU) } \\
15 \text { mins: AI Systems for Inclusive Education \& Disabilities } \\
\text { (expert presentation, Amrita) } \\
15 \text { mins: AI and Privacy in the context of Accessibility and } \\
\text { Inclusivity (expert presentation, Amrita) } \\
45 \text { mins: Team activity } \# 2 \text { in breakout rooms } \\
\text { Design one future scenario: } \\
\text { Where accessible or inclusive AI is used } \\
\text {-Consider how one or more of the challenges identified in the } \\
\text { team activity } \# 2 \text { were alleviated with this design } \\
\text { scenario/solution } \\
\text {-Reflect on what needs to change to realize this scenario in the } \\
\text { future. } \\
15 \text { mins: Team presentation to the larger group } \\
15 \text { mins: Workshop wrap-up and next steps (TAU) }\end{array}$ \\
\hline
\end{tabular}

and empowering AI, and case studies and methods for designing for the future. In the hands-on activities, participants will work in teams of 3-4 to collaboratively and conceptually define what is Accessible and Inclusive AI, and what are the current challenges towards it. In the second team activity, participants work in the same teams to design one future scenario which includes an accessible or inclusive AI, and how and why one or more of the challenges were alleviated. The teams will then briefly present their designs to the larger group. Team discussions are moderated by the workshop organizers. Therefore, through the teamwork, participants will reimage what it means for AI to be accessible, inclusive, and empowering, and discuss opportunities for the future. Each team will be provided pens, papers, and other material to write and draw during the activities if the activity is face-to-face.

If the activity is online, the participants are provided an online environment for the work, with the environment set up beforehand by the organizers. Live captioning and the option to participate via chat is provided to participants to keep the workshop accessible.

\section{CALL FOR PARTICIPATION}

In this full-day workshop, join us to formulate a shared vision for accessible and inclusive AI and identify the challenges and opportunities towards this vision. We invite students, researchers, experts, and practitioners, interested in $\mathrm{AI}$ from the perspectives of inclusion and accessibility, and through those also empowerment, to collaboratively explore and reimagine AI futures. We also invite researchers who have an interest in critical design, design fiction, speculative design, and other future oriented design approaches.

Please submit a short position paper (in the new ACM template, max 3 pages, as a .docx file) by filling in the form on the workshop website. In the paper, please mention

- author names and affiliations,

- a short description of your research interests, driving values and motivations, goals, and your vision for the future of your own research,
- a short mention of how your work addresses the workshop topic,

- a short paragraph on your own vision and goals for AIAI, and

- how do you see that it would be possible to reach that vision? $\circ$ who are the ones whose actions are needed for the vision to come true?

$\circ$ and how it is possible to measure that the vision comes true?

- Optionally, you can provide an overview of a study, project, or research paper that you are most proud of.

For more information, please visit the workshop website: https: //interact.oulu.fi/researcherstoolbox

\section{INSTRUCTORS}

Dr. Marianne Kinnula is an Associate Professor of humancentred design and digitalization in INTERACT Research Unit in University of Oulu. Her research focus is on how technology changes our everyday lives in many ways, at society level, organizational level, as well as at individual level. Dr. Netta Iivari is a Professor in Information Systems and research unit leader of INTERACT Research Unit in University of Oulu. Her research addresses critical design, critical Making, empowerment and inclusion. Dr. Sumita Sharma is a post-doc researcher at the INTERACT Research Unit at the University of Oulu. Her research work focuses on designing inclusive and collaborative technology for marginalized users.

The INTERACT Research Unit has a strong track on teaching and research on human-centered design and digitalization. Particularly strong has been research on empowerment, inclusion and diversity as regards digital technology and its design. INTERACT has conducted research on the topics for well over ten years with a number of externally funded research projects, doctoral theses and high-quality scientific publications. INTERACT has explored empowering and inclusive design approaches as well as how to increase diversity in the IT workforce. Currently, INTERACT research projects inquire novel empowering and critical design approaches 
with schoolchildren (Academy of Finland and H2020 funded project) as well as with different kinds of marginalized groups and communities (ESF funded project) as well as societal impact of novel technologies (Academy of Finland funded GenZ project). INTERACT has studied ethical aspects of AI and is collaborating with CogniAble on accessibility and AI, devising a framework for human agency and oversight in AI, as outlined in the EU directives for Trustworthy AI (EU Guidelines). CogniAble is a screening and therapy-based mobile AI based application for parents of young children with autism to access tailored and targeted behavior and language interventions.

Dr. Grace Eden is an Assistant Professor in the Department of Human-Centered at Indraprastha Institute of Information Technology, Delhi (IIITD). She conducts teaching and research with a focus on interaction design and user experience to understand the effects of technology on human behaviour, communication and social interaction.

Dr. Markku Turunen is a professor of Interactive Technology in the Faculty of Information Technology and Communication Sciences at Tampere University, Finland (TAU). He is leading a research group on Pervasive Interaction and a M.Sc. programme on Sustainable Digital Life. Recently, he established with his colleagues a research and education unit on accessibility entitled "TACCU TACCU -Tampere Accessibility Unit” (https://research.tuni.fi/taccu/ ) .

TAU has decades of experience and expertise in accessibility working with users with special needs in Finland and India. In Finland, this includes e.g. assistive technology tools for people with visual, physical and cognitive impairments. In India, this includes a pilot of a kiosk-style interactive health information system for rural Assamese women, and developing suitable interactive content for classroom learning employing gesture interaction for children with autism to learn and interact socially. Further, TAU is working towards a one-of-a-kind course on Accessibility (15 ECTS), where the first course on Fundamentals of Accessibility (5 ECTS) was conducted in August 2020 as a part of its summer school.

Dr. Krishnashree Achuthan is the Dean of Post Graduate Programs at Amrita Vishwa Vidyapeetham and Dr. Prema Nedungadi is Director of AmritaCREATE, an award-winning, research center that focuses on multidisciplinary research at the intersection of Computer Science, Education, Social Sciences. Amrita has a strong emphasis on Artificial Intelligence and Machine Learning with 400+ co-authored papers, 9 books and several patents on the topic, and 6 Masters programs and Phd programs in Artificial Intelligence, Data Sciences and Machine Learning. Amrita has an active Livein-Labs ${ }^{\circledR}$ program (experiential learning), Cybersecurity Systems and Networks and Next Generation Amrita Technology Business Incubator. Amrita's core vision lies in providing education for life grounded in intellectual social responsibility through compassion driven research that will translate into a positive global impact.

Tero Avellan is a doctoral researcher in Interactive Technology in the Faculty of Information Technology and Communication Sciences at Tampere University, Finland (TAU). His research focuses on the various socio-technical aspects of accessible and assistive technology in the society of the future.

Biju Thankachan is a doctoral candidate in Interactive Technology at Tampere University, Finland. His research focuses on designing and developing interactive solutions for emergent and remote users. Currently, he is working on exploring the usage of Artificial Intelligence among small and medium enterprises in Tampere. His academic research is on designing and developing Voice User Interfaces in Semi-Public Spaces, especially for the elderly.

Rucha Tulaskar is a doctoral researcher in Interactive Technology from faculty of Information Technology and Communication Sciences from Tampere University (TAU). Her work focuses on exploring immersive technology like social Virtual Reality for remote higher education.

\section{ACKNOWLEDGMENTS}

This work is funded by the Finnish National Agency for Education Asia Programme (in Finland at Tampere University and University of Oulu, and in India at Indraprastha Institute of Information Technology Delhi and Amrita Vishwa Vidyapeetham) as well as the Academy of Finland (Grant \#324685, Make-a-Difference project) at the University of Oulu. This research is connected to the GenZ project, a strategic profiling project in human sciences at the University of Oulu. The project is supported by the Academy of Finland (Grant \#318930) and the University of Oulu.

\section{REFERENCES}

[1] Anupam Chander. 2017. THE RACIST ALGORITHM? Michigan Law Review 115, 6 (2017), 1023-1045.

[2] Tero Avellan, Sumita Sharma, and Markku Turunen. 2020. AI for all: defining the what, why, and how of inclusive AI. In Proceedings of the 23rd International Conference on Academic Mindtrek (AcademicMindtrek '20). Association for Computing Machinery, New York, NY, USA, 142-144.

[3] Jeffrey Dastin. 2018. Amazon scraps secret AI recruiting tool that showed bias against women. San Fransico, CA: Reuters.

[4] Dunne, A., \& Raby, F. (2013). Speculative everything: design, fiction, and social dreaming. MIT press.

[5] EU Guidelines: Ethics guidelines for trustworthy AI, EU directive. Retrieved on August 12th 2020 from https://ec.europa.eu/digital-single-market/en/news/ ethics-guidelines-trustworthy-ai

[6] IIIT-Delhi: HCAI course project work in ACM conference on Design of Interactive Systems. Retrieved August 13th from https://blog.iiitd.ac.in/thinking-out-of-thebox-course-projects-by-iiit-delhi-students/

[7] Iivari, Netta. "Empowering children to make and shape our digital futures-from adults creating technologies to children transforming cultures." The International Journal of Information and Learning Technology (2020).

[8] Netta Iivari, Marianne Kinnula, Tonja Molin-Juustila, and Leena Kuure. "Exclusions in social inclusion projects: Struggles in involving children in digital technology development." Information Systems Journal 28, no. 6 (2018): 10201048.

[9] Marianne Kinnula, Netta Iivari, Tonja Molin-Juustila, Eino Keskitalo, Topi Leinonen, Eetu Mansikkamäki, Toni Käkelä, and Martti Similä. "Cooperation, Combat, or Competence Building-What Do We Mean When We Are 'Empowering Children'in and through Digital Technology Design?." In ICIS. 2017.

[10] Atte Kinnula, Marianne Kinnula, and Harko Verhagen. "New Technologies for the Good of Whom? Whose Values Win?." Computing Professionals for Social Responsibility: The Past, Present and Future Values of Participatory Design (2020).

[11] Meredith Ringel Morris. 2020. AI and Accessibility: A discussion of ethical considerations. Commun. ACM 63, 6 (May 2020), 35-37. DOI:https://doi.org/10.1145/ 3356727

[12] Sharma, Sumita, Krishnaveni Achary, Marianne Kinnula, Netta Iivari, and Blessin Varkey. "Gathering garbage or going green? shifting social perspectives to empower individuals with special needs." In Proceedings of the Interaction Design and Children Conference, pp. 311-322. 2020

[13] Vainionpää, Fanny, Marianne Kinnula, Netta Iivari, and Tonja Molin-Juustila. "GIRLS' CHOICE - WHY WON’T THEY PICK IT?" (2019). European Conference on Information Systems (ECIS)

[14] Voros, J. (2017). Big History and Anticipation: using big history as a framework for global foresight. Handbook of anticipation: Theoretical and applied aspects of the use of future in decision making.

[15] James Zou and Londa Schiebinger. 2018. AI can be sexist and racist - it's time to make it fair. Nature 559, 7714 (jul 2018), 324-326. 